\title{
Attenuation of Diastolic Heart Failure and Life-Threatening Ventricular Tachyarrhythmia After Peripheral Blood Stem Cell Transplantation Combined With Cardioverter-Defibrillator Implantation in Myeloma-Associated Cardiac Amyloidosis
}

\author{
Hiroyuki Yaoita, MD; Masumi Iwai-Takano, MD; Kazuei Ogawa, MD; Hitoshi Suzuki, MD; \\ Kazuko Akutsu, MD; Hideyoshi Noji, MD; Yoshiyuki Kamiyama, MD; \\ Satoshi Kimura, MD; Hideki Ohtake, MD; \\ Toshiyuki Ishibashi, MD; Yukio Maruyama, MD
}

\begin{abstract}
A patient had multiple myeloma and associated cardiac amyloidosis, which caused diastolic dysfunction and recurrent ventricular fibrillation. After implantation of a cardioverter-defibrillator (ICD), the patient underwent autologous peripheral blood stem cell transplantation (PBSCT). The life-threatening arrhythmias, such as ventricular fibrillation, disappeared, and diastolic dysfunction assessed by quantitative gated single photon emission computed tomography and Doppler echocardiography improved 7 months later. This may be the first report to document improvement of both a lethal rhythm disorder and diastolic dysfunction by PBSCT following ICD implantation in a case of cardiac amyloidosis associated with multiple myeloma. (Circ J 2008; 72: 331-334)
\end{abstract}

Key Words: Amyloid; Arrhythmia; Cardiomyopathy; Heart failure; Transplantation

$\mathbf{C}$ ardiac amyloidosis caused by extracellular deposition of amyloid light-chain (AL) associated with multiple myeloma is characterized by progressive heart failure (HF), especially with diastolic dysfunction and sudden death because of lethal arrhythmias such as ventricular fibrillation, resulting in a poor prognosis! Although little evidence is available with regard to the most appropriate management for cardiac amyloidosis, there has been a paper that documented improvement of cardiac function, especially diastolic dysfunction, after chemotherapy and stem cell transplantation for multiple myeloma ${ }^{2}$ and primary systemic amyloidosis $3^{3-5}$ However, it remains unknown whether stem cell transplantation for multiple myeloma prevents lifethreatening ventricular arrhythmias and improves HF, both of which are manifestations of cardiac amyloidosis. We describe a patient with diastolic HF and recurrent ventricular fibrillation, because of cardiac amyloidosis associated with multiple myeloma, who was pretreated with an implantable cardioverter-defibrillator (ICD), followed by chemotherapy and subsequent autologous peripheral blood stem cell transplantation (PBSCT).

(Received March 14, 2007; revised manuscript received September 4, 2007; accepted September 14, 2007)

First Department of Internal Medicine, Fukushima Medical University, Fukushima, Japan

Mailing address: Masumi Iwai-Takano, MD, First Department of Internal Medicine, Fukushima Medical University, Hikarigaoka 1, Fukushima 960-1295, Japan. E-mail: masumi @ fmu.ac.jp All rights are reserved to the Japanese Circulation Society. For permissions, please e-mail: cj@j-circ.or.jp

\section{Case Report}

In November 2005, a 68-year-old woman was admitted to another hospital for shortness of breath and pretibial edema. Diagnosed with left ventricular (LV) hypertrophy and diastolic dysfunction, she was given supplementary oxygen and oral diuretics. Two weeks later, she suddenly developed ventricular fibrillation, which was reversed by external electrical defibrillation. Because an IgGK type monoclonality on serum immuno-electrophoresis, urinary Bence-Jones protein ( $k$ type), and osteolytic lesions of the ribs were detected, bone marrow biopsy was performed. Plasma cells (CD20-negative, CD138-positive, Ig Ck typepositive) were detected in $18.2 \%$ of the total nuclear cell counts. In December 2005, she developed non-sustained ventricular tachycardia with syncope and because cardiac amyloidosis was suspected, she was referred to us for further treatment in January 2006. On admission, her cardiothoracic ratio was $65 \%$, and HF status was grade III of the New York Heart Association (NYHA) functional classification, and profile B of Nohria's classification? Two-dimensional Doppler and tissue Doppler echocardiography showed restrictive patterns of LV function [3.22 in E/A (the ratio of transmitral early diastolic filling wave and the filling wave related to atrial contraction), $150 \mathrm{~ms}$ in deceleration time, 19.2 in E/E' (ratio of transmitral early diastolic filling wave and mitral annular early velocity); ${ }^{8}$ Table 1]. Quantitative ECG-gated single photon emission computed tomography $(\mathrm{QGS})^{9}$ on ${ }^{99 \mathrm{~m} T c}$ myocardial perfusion imaging at rest showed an unusually gentle slope of the early diastolic filling on the LV time-volume curve (Fig 1). The results of both imaging modalities indicated the presence of severe diastolic dysfunction. Cardiac catheterization data re- 
Table 1 Laboratory Data, Doppler and Tissue Doppler Echocardiography, and QGS on Myocardial Perfusion Imaging at Rest in the Clinical Course

\begin{tabular}{|c|c|c|c|c|}
\hline & Before PBSCT & 7 weeks & 7 months & $\begin{array}{l}10 \text { months } \\
\text { after PBSCT }\end{array}$ \\
\hline $\operatorname{Ig} G(m g / d l)$ & 1,832 & 885 & 1,251 & 1,374 \\
\hline$C R P(m g / d l)$ & 3.3 & 0.1 & 0.1 & 0.1 \\
\hline Albumin $(g / d l)$ & 2.7 & 3.7 & 4.1 & 3.9 \\
\hline Hemoglobin $(\mathrm{g} / \mathrm{dl})$ & 10.8 & 11.7 & 10.5 & 10.4 \\
\hline$B N P(p g / m l)$ & 1,200 & 659 & - & 90 \\
\hline \multicolumn{5}{|c|}{ Doppler and tissue Doppler echocardiography } \\
\hline E/A (ratio) & 3.22 & 1.95 & 1.28 & 0.88 \\
\hline$D C T(m s)$ & 150 & 139 & 160 & 173 \\
\hline$E / E^{\prime}($ ratio $)$ & 19.2 & - & 10.5 & 9.9 \\
\hline$L V W T(\mathrm{~mm})$ & 16 & 16 & 16 & 16 \\
\hline \multicolumn{5}{|l|}{$Q G S$} \\
\hline $\operatorname{LVEDVI}\left(\mathrm{ml} / \mathrm{m}^{2}\right)$ & 49 & 47 & 58 & \\
\hline $\operatorname{LVEF}(\%)$ & 0.46 & 0.42 & 0.55 & \\
\hline \multicolumn{5}{|l|}{ Therapies } \\
\hline & $I C D \quad D E X$ & $M P B S C T$ & & \\
\hline
\end{tabular}

QGS, quantitative gated single photon emission computed tomography; PBSCT, peripheral blood stem cell transplantation; BNP, $B$-type natriuretic peptide; DCT, deceleration time; LVWT, left ventricular septal wall thickness; LVEF, left ventricular ejection fraction; ICD, implantation of a cardioverter-defibrillator; DEX, dexamethasone; L-PAM; melphalan.

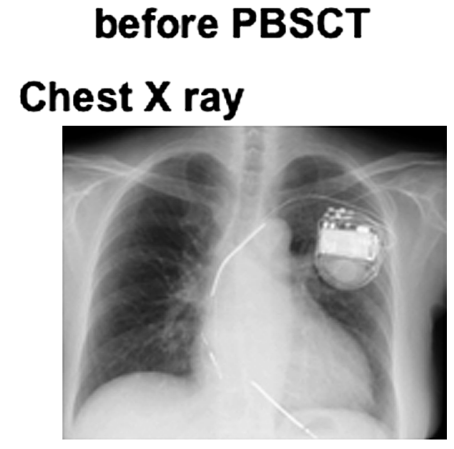

\section{7 weeks after PBSCT}

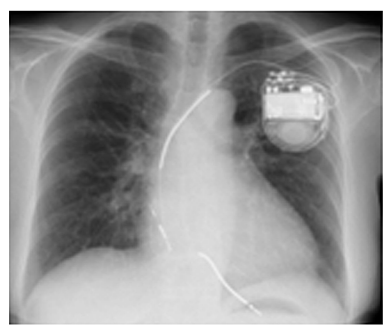

Transmitral Doppler echocardiography
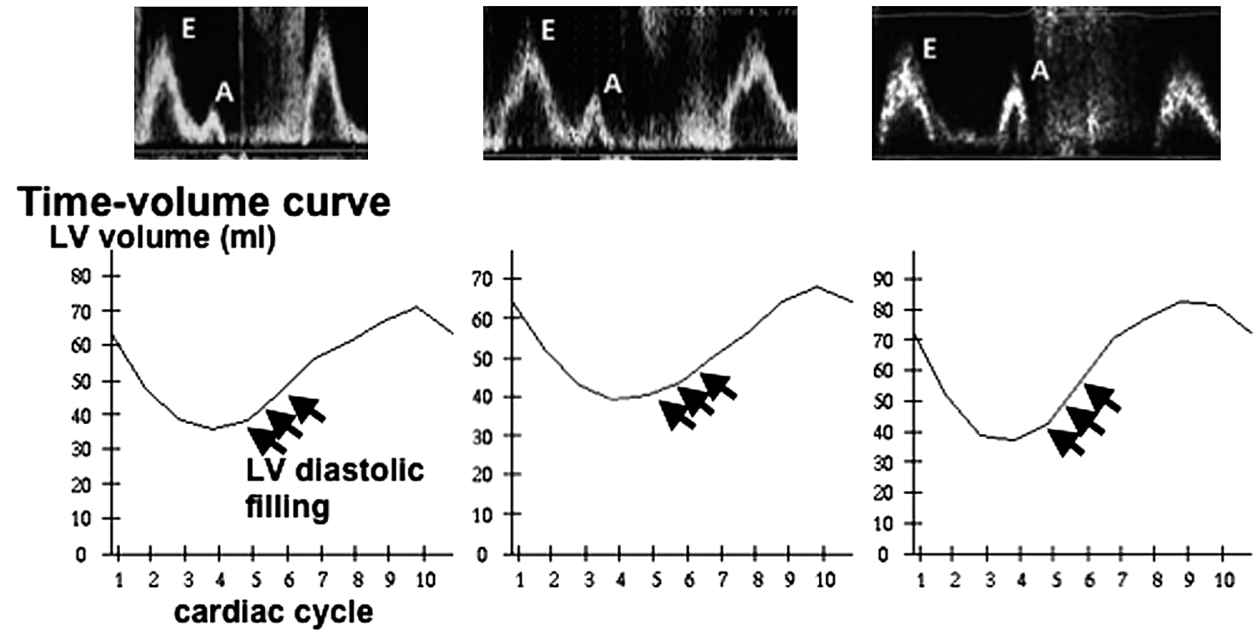

Fig 1. Chest X ray, the time-volume curves on quantitative gated single photon emission computed tomography at rest, and the transmitral early diastolic filling wave and the filling wave because of atrial contraction on Doppler echocardiography.

vealed no coronary obstructive lesions and preservation of systolic function, but there was severe diastolic cardiac dysfunction (LV peak systolic pressure/end-diastolic pressure $87 / 17 \mathrm{mmHg}$, heart rate 61 beats $/ \mathrm{min}$, cardiac index $1.53 \mathrm{~L} \cdot \mathrm{min}^{-1} \cdot \mathrm{m}^{-2}$, pulmonary arterial peak systolic pressure/diastolic pressures $33 / 13 \mathrm{mmHg}$, and mean right atrial pressure $6 \mathrm{mmHg}$ ) and LV endomyocardial biopsy specimens showed extracellular deposition of AL-type amyloid fibrils in which Congo red-staining was broken down by $\mathrm{KMnO}_{4}$ oxidation.

In February 2006, she was diagnosed as having multiple myeloma according to the Southwest Oncology Group 
(SWOG) classification ${ }^{10}$ and to be in stage II of the International Prognostic Index (serum albumin $2.7 \mathrm{~g} / \mathrm{dl}, \beta_{2}$-microglobulin $2.49 \mu \mathrm{g} / \mathrm{ml}$ ). She developed ventricular fibrillation again, which required cardiopulmonary resuscitation, and an ICD ${ }^{1-13}$ (Maximo DR ${ }^{\mathrm{TM}}$, Medtronic, Minneapolis, MN, USA) (Table 1). HF symptoms were attenuated by treatment with oral diuretics and intravenous recombinant human atrial natriuretic peptide (carperitide) ${ }_{14}^{4}$ although the severe mechanical diastolic failure documented by 2-dimensional Doppler echocardiography persisted (Fig 1). In the same month, she developed ventricular fibrillation, which was promptly defibrillated by the ICD. After pretreatment with dexamethasone $40 \mathrm{mg}$ once daily for 4 days, peripheral blood stem cells were collected by leuko-apheresis after mobilization using granulocyte colony stimulating factor. After high-dose dexamethasone $(40 \mathrm{mg} /$ day) treatment for 4 days again and melphalan $100 \mathrm{mg} /$ day for 2 days, autologous PBSCT was performed in early April (Table 1). In May, her HF status had improved to NYHA functional class IIm, and she was discharged. In June 2006, the absence of detectable monoclonal protein was confirmed in serum immunofixation electrophoresis. There was no recurrence of life-threatening arrhythmias or defibrillation events by the ICD after autologous PBSCT. In October 2006 (approximately 7 months after PBSCT) on regular admission to the outpatient clinic, her HF status was improving to class IIs of the NYHA and profile A of Nohria. By Doppler and tissue Doppler echocardiography, the marked increases in E/A and E/E' had normalized, and the pattern of the LV timevolume curve on QGS showed rapid filling, all of which indicated improvement of the severe LV diastolic dysfunction (Fig 1). Ten months after PBSCT, the laboratory data remained stable, there were no findings of inappropriate shocks by the ICD, and the E/A and E/E' had further decreased, confirming continued improvement of diastolic function.

\section{Discussion}

The present case suggests that autologous PBSCT is a useful therapy for cardiac involvement of amyloidosis, even if the patient has life-threatening arrhythmias. In addition to remission of multiple myeloma, such problematical arrhythmias and diastolic HF could be ameliorated by autologous PBSCT. Although we need to follow the clinical course of the present patient long-term, the data documented here suggest that cardiac amyloidosis associated with multiple myeloma may not be necessarily therapeutically barren.

Twenty-eight weeks after PBSCT, some may note the slightly increased LV end-diastolic volume index on QGS, despite improvement of LV diastolic dysfunction and a reduction of the cardiothoracic ratio. It is possible to speculate that improvement of $\mathrm{LV}$ diastolic compliance served to bring in more blood in the end-diastolic phase because the LV ejection fraction was rather increased. A reduction in the cardiothoracic ratio at that time may reflect a reduction of volume load on the right heart, as assumed from the diminished estimated pulmonary arterial pressure $(0$ on the trans-tricuspid valve pressure gradient).

Our results showed that diastolic dysfunction can improve without regression of LV wall thickness, although this may occur in the future. The mechanism for this favorable response to treatment remains unclear. It has been reported that amyloid light chains increase intracellular free radicals, alter calcium handling, and cause impairment of relaxation as well as contractility in isolated cardiomyocytes ${ }^{15}$ Although we could not perform repeated histological examinations after PBSCT, the production of amyloid light chains may decrease in conjunction with the complete hematologic response and this may cause the improvement of cardiac function. Nakamura et $\mathrm{al}^{2}$ reported a case of cardiac amyloidosis, because of multiple myeloma, which was successfully treated with chemotherapy, after which the pseudonormalized E/A decreased, the thick LV wall regressed, and the LV ejection fraction increased over 3 years. That was the first report to clearly document reversal of diastolic dysfunction by chemotherapy in a long-term observation of cardiac amyloidosis.

Itoh et $\mathrm{al}^{16}$ reported a case of cardiac amyloidosis with ventricular fibrillation attacks in which an ICD was implanted ${ }^{16}$ During the 2-month observation period until death from systemic amyloidosis, 5 episodes of ventricular fibrillation were successfully treated by the ICD. Reports by Itoh et $\mathrm{al}^{16}$ and others ${ }^{13}$ suggest not only that ICD therapy may be applicable for preventing arrhythmic death caused by cardiac amyloidosis, but also that rapid progression of systemic amyloidosis, irrespective of arrhythmic events, is another determinant of the patient's prognosis.

Thus, preserved LV systolic function, compensated congestive HF and the absence of life-threatening arrhythmia may enable chemotherapy/PBSCT to be performed in their patient and some studies 2,5 because of the prevention from cardiac death during therapy.

The present case had recurrent life-threatening ventricular arrhythmias, in addition to marked diastolic HF. Although the risk of sudden death from such arrhythmias was reduced by implantation of the ICD, the prognosis of this patient with cardiac amyloidosis was assumed to be still poor because of plausible progression of the diastolic HF $!^{17}$ Therefore, after consultation and discussion among the hematologists, cardiologists and the patient, we reached the decision to perform autologous PBSCT, making this, as far as we know, the first case of a patient in whom significant improvement of remarkable diastolic HF and the reduction of the risk of arrhythmic sudden death was documented after PBSCT, possibly owing to recent advances in anti-HF and anti-arrhythmic device therapies.

\section{References}

1. Cueto-Garcia L, Tajik AJ, Kyle RA, Edwards WD, Greipp PR, Callahan JA, et al. Serial echocardiographic observations in patients with primary systemic amyloidosis: An introduction to the concept of early (asymptomatic) amyloid infiltration of the heart. Mayo Clin Proc 1984; 59: 589-597.

2. Nakamura M, Satoh M, Kowada S, Satoh H, Tashiro A, Sato F, et al. Reversible restrictive cardiomyopathy due to light-chain deposition disease. Mayo Clin Proc 2002; 77: 193-196.

3. Dispenzieri A, Kyle RA, Lacy MQ, Therneau TM, Larson DR, Plevak MF, et al. Superior survival in primary systemic amyloidosis patients undergoing peripheral blood stem cell transplantation: A case-control study. Blood 2004; 103: 3960-3963.

4. Dispenzieri A, Gertz MA, Kyle RA, Lacy MQ, Burritt MF, Therneau TM, et al. Prognostication of survival using cardiac troponins and $\mathrm{N}$-terminal pro-brain natriuretic peptide in patients with primary systemic amyloidosis undergoing peripheral blood stem cell transplantation. Blood 2004; 104: 1881-1887.

5. Skinner M, Sanchorawala V, Seldin DC, Dember LM, Falk RH, Berk JL, et al. High-dose melphalan and autologous stem-cell transplantation in patients with AL amyloidosis: An 8-year study. Ann Intern Med 2004; 140: 85-93.

6. Nohria A, Tsang SW, Fang JC, Lewis EF, Jarcho JA, Mudge GH, et al. Clinical assessment identifies hemodynamic profiles that predict outcomes in patients admitted with heart failure. J Am Coll Cardiol 2003; 41: 1797-1804. 
7. Klein AL, Hatle LK, Taliercio CP, Taylor CL, Kyle RA, Bailey KR, et al. Serial Doppler echocardiographic follow-up of left ventricular diastolic function in cardiac amyloidosis. J Am Coll Cardiol 1990; 16: $1135-1141$

8. Samad BA, Olson JM, Alam M. Characteristics of left ventricular diastolic function in patients with systolic heart failure: A Doppler tissue imaging study. J Am Soc Echocardiogr 2005; 18: 896-900.

9. Germano G, Erel J, Lewin H, Kavanagh PB, Berman DS. Automatic quantitation of regional myocardial wall motion and thickening from gated technetium-99m sestamibi myocardial perfusion single-photon emission computed tomography. J Am Coll Cardiol 1997; 30: 1360 1367.

10. Rajkumar SV, Kyle RA. Multiple myeloma: Diagnosis and treatment. Mayo Clin Proc 2005; 80: 1371-1382.

11. Wright BL, Grace AA, Goodman HJ. Implantation of a cardioverterdefibrillator in a patient with cardiac amyloidosis. Nat Clin Pract Cardiovasc Med 2006; 3: 110-114.

12. Tanno K, Miyoshi F, Watanabe N, Minoura Y, Kawamura M, Ryu S, et al, MADIT II. The Multicenter Automatic Defibrillator Implanta- tion Trial: Are the MADIT II criteria for ICD implantation appropriate for Japanese patients? Circ J 2005; 69: 19-22.

13. Richter S, Duray G, Gronefeld G, W Israel C, H Hohnloser S. Prevention of sudden cardiac death: lessons from recent controlled trials. Circ J 2005; 69: 625-629.

14. Tsuneyoshi H, Nishina T, Nemoto T, Kanemitsu H, Kawakami R, Unimonh $\mathrm{O}$, et al. Atrial natriuretic peptide helps prevent late remodeling after left ventricular aneurysm repair. Circulation 2004; 110(Suppl 1): II-174-II-179.

15. Brenner DA, Jain M, Pimentel DR, Wang B, Connors LH, Skinner $\mathrm{M}$, et al. Human amyloidogenic light chains directly impair cardiomyocyte function through an increase in cellular oxidant stress. Circ Res 2004; 94: $1008-1010$.

16. Itoh M, Ohmori K, Yata K, Wada H, Tsuchiya T, Okahashi N, et al. Implantable cardioverter defibrillator therapy in a patient with cardiac amyloidosis. Am J Hematol 2006; 81: 560-561.

17. Parikh S, de Lemos JA. Current therapeutic strategies in cardiac amyloidosis. Curr Treat Options Cardiovasc Med 2005; 7: 443448. 